\title{
Prof. Dr. Dr. h.c. Volker Storch zum 65. Geburtstag gewidmet
}

\author{
Henner Hollert • Thomas Braunbeck (Herausgeber)
}

Eingegangen: 13. Januar 2009/Akzeptiert: 24. Februar 2009/Online veröffentlicht: 20. März 2009

(C) Springer-Verlag 2009

Dieses Sonderheft ist Prof. Dr. Dr. h. c. Volker Storch gewidmet, der im Frühjahr 2009 emeritiert wird und in den letzten 3 Jahrzehnten als Leiter des Instituts für Zoologie I der Universität Heidelberg die deutsche Zoologie, die Biodiversitäts- und die Umweltforschung maßgeblich geprägt hat. In diesem Sonderband kann keineswegs das Wirken von Volker Storch in vollem Umfang gewürdigt werden. Vielmehr ist es das erklärte Ziel dieses Sonderbandes, einen - sicherlich subjektiven - Überblick über Forschungsschwerpunkte in den Bereichen Ökotoxikologie, Angewandte Zoologie und Biodiversitätsforschung zu geben, die in den letzten 15 bis 20 Jahren am Heidelberger Institut für Zoologie entstanden sind. Zum Teil haben diese Forschungsschwerpunkte, u. a. durch die akademischen Schüler von Volker Storch, sehr weite Verbreitung erfahren (z. B. biologische Stechmückenbekämpfung, Fischnetz Schweiz, Entwicklung von Alternativmethoden zu Toxizitätstests).

Volker Storch studierte ab 1963 an der Universität Kiel und wurde bereits im 11. Semester (!) 1968 mit einer vergleichenden Arbeit über die Muskulatur von Polychaeten bei Prof. Remane mit „summa cum laude“ promoviert. Nach einer Assistentenzeit bei Prof. Schuster habilitierte

Prof. Dr. Dr. h. c. Volker Storch zum 65. Geburtstag gewidmet.

H. Hollert $(\bowtie)$

Lehr- und Forschungsgebiet für Ökosystemanalyse,

Institut für Umweltforschung (Biologie V),

Rheinisch-Westfälische Technische Hochschule Aachen,

Worringerweg 1, 52074 Aachen, Deutschland

E-Mail: henner.hollert@bio5.rwth-aachen.de

T. Braunbeck

Aquatische Ökologie und Toxikologie,

Institut für Zoologie, Universität Heidelberg,

Im Neuenheimer Feld 230, 69120 Heidelberg, Deutschland

E-Mail: braunbeck@zoo.uni-heidelberg.de sich Storch 1971 im Fach Zoologie. Neben der Lehrtätigkeit am Kieler Institut hielt er als Professor Vorlesungen in Hamburg und übernahm eine Kurzzeitdozentur auf den Philippinen. 1978 erhielt Volker Storch im Alter von 35 Jahren einen Ruf auf den Lehrstuhl für Morphologie und Ökologie der Tiere an die Universität Heidelberg, den er bis zu seiner Emeritierung inne hatte. Im Jahr 2004 wurde ihm die Ehrendoktorwürde der Universität Greifswald verliehen. Prof. Storch hat über 250 Originalarbeiten publiziert, von denen mehr als 150 in ISI Web of Science gelistet sind und insgesamt über $1800 \mathrm{mal}$ zitiert wurden.

Wahrhaft herausragend sind die Leistungen von Volker Storch als Lehrbuchautor. 1971 verfasste er auf Remanes Wunsch als „Gesellenprüfung“ ein Bändchen über „Meeresborstenwürmer“. 1972 wurde die erste Auflage des „Kurzen Lehrbuchs der Zoologie“ gemeinsam mit Remane und Welsch publiziert, das inzwischen in der achten Auflage vorliegt. Es folgten 1973 ein Taschenbuch „Evolution - Tatsachen und Probleme der Abstammungslehre" und eine „Einführung in Cytologie und Histologie der Tiere“; weiterhin ab 1976 die „Systematische Zoologie“, ab 1991 „Kükenthals Leitfaden für das Zoologische Praktikum“ und ab 2001 eine „Evolutionsbiologie“. Insgesamt sind seine Bücher nunmehr in 8 Sprachen erschienen.

Für alle Erstautoren und die meisten Mitautoren dieses Sonderbandes ist Volker Storch akademischer Lehrer und Mentor, der durch seine Weitsicht den eigenen Forschungsschwerpunkten immer neue Perspektiven aufzeigen konnte. Obgleich seine eigene Forschung zu größten Teilen in den Bereichen der vergleichenden Ultrastrukturforschung angesiedelt ist, war ihm die Umwelt- und Biodiversitätsforschung an seinem Institut immer ein sehr wichtiges Anliegen. So entstand in den 80er Jahren eine vergleichende ökotoxikologische Ultrastrukturforschung, für die Storch das Motto „Zelle und Umwelt“ geprägt hat. Diese Arbei- 
ten haben experimentell in breitem Umfang Reaktionen gegenüber Umwelteinflüssen auf zellulärer bzw. histologischer Ebene untersucht (z. B. Wirkung von Nahrungsentzug, Nahrungsqualität, diversen Stressoren: Temperatur, Noxen).

Helmut Segner und Rita Triebskorn geben in ihren Beiträgen Morphologische Parameter als Biomarker in der Ökotoxikologie: natürliche und Schadstoff-induzierte Variabilität sowie Schnecken unter Stress - Gastropoden als Modelle in Ökophysiologie und Ökotoxikologie einen Überblick über Ausschnitte aus diesem Forschungsfeld. Diese Arbeitsrichtung hat auch weit in die biochemisch-molekulare Ebene hineingewirkt, beeinflusst massiv die Einschätzung von Biotests, hat zu Untersuchungen von alternativen nichtorganismischen Biotests geführt und wirkt noch weiter.

Im Beitrag von Heinz-R. Köhler wird in diesem Kontext die Rolle von Stressproteinen bei der Anpassung an Umweltbedingungen behandelt.

Neben dem Schwerpunkt der Entwicklung von akuten und Mechanismus-spezifischen Biotestverfahren wurden am Zoologischen Institut auch Sedimenttests entwickelt; mit ihnen partikulär-gebundene Schadstoffe wurden auf ihre adversen Wirkungen hin untersucht. Henner Hollert et al. geben in diesem Kontext einen Überblick über Strategien zur Sedimentbewertung.

Berichte über Fischrückgänge bzw. Fischpopulationen mit ungewöhnlicher Populationszusammensetzung in zahlreichen Flusssystemen in Europa und Nordamerika stehen in scharfem Kontrast zu Analysen der Gewässergüte, die für die letzten Jahre eindeutig eine Verbesserung belegen. Patricia Burkhardt-Holm geht in ihrem Beitrag auf Basis von Untersuchungen aus dem Schweizer Projekt „Fischnetz“ mit seinen 77 Teilprojekten der Frage nach, ob ,Climate change und Bachforellenrückgang im Zusammenhang stehen.

Steffen Keiter et al. stellen vor, wie in umfassenden Weight-of-Evidence-Analysen in den Jahren 2003 bis 2008 der Fischrückgang in der oberen Donau untersucht wurde.

Der Beitrag von Thomas Braunbeck et al., Fischpopulationen unter Stress - das Beispiel des Unteren Neckars, zeigt, wie Biotests zur Mechanismus-spezifischen Toxizität (hier: Gentoxizität und Teratogenität) mit vergleichenden ökotoxikologischen Ultrastrukturuntersuchungen kombiniert werden können, um Hinweise auf Mechanismen zu finden, die den Defiziten in der Zusammensetzung der Fischpopulationen zugrunde liegen könnten.

Im Bereich der angewandten Zoologie ist Volker Storch vor allem die biologische Stechmückenbekämpfung mit
Toxinen des Bacillus thuringiensis ssp. israelensis (Bti) als umweltverträgliche Alternative zu vielen synthetischen Pestiziden ein großes Anliegen gewesen. Das aktuelle Grundlagenwissen wurde in diesem Bereich zur Bekämpfung von Stechmücken im Oberrhein und auch zur Bekämpfung von Infektionskrankheiten in Entwicklungsländern eingesetzt. Nobert Becker gibt in seinem Beitrag einen Überblick über die Rolle der Globalisierung und Klimaveränderung auf die Entwicklung von Stechmücken und von ihnen übertragenen Krankheiten in Zentral-Europa.

Schließen soll dieses Sonderheft mit dem Thema, das Prof. Storch vermutlich am meisten am Herzen liegt: Erforschung und Schutz der Biodiversität. Er hat sich viele Jahre intensiv mit dem Studium der marinen Biodiversität in verschiedenen Evertebratentaxa beschäftigt (vgl. Storch und Wehe 2007) und im letzten Jahrzehnt unter anderem die großen deutschen Meeresforschungsinstitute und Universitäten mit marinen Forschungsschwerpunkten für die DFG und den Wissenschaftsrat begutachtet. Zurzeit sitzt Storch dem Wissenschaftlichen Beirat des Deutschen Zentrums für Marine Biodiversitätsforschung, Wilhelmshaven vor, dessen Gründung seinem Einsatz mit zu verdanken ist, und dem Wissenschaftlichen Beirat des Senckenberg Museums und ist Mitglied im Beirat der Sächsischen Naturkundlichen Museen. Das Sonderheft soll daher mit dem Thema schließen, mit dem die wissenschaftliche Laufbahn von Prof. Volker Storch begonnen hat: Thomas Wehe stellt in seinem Beitrag vor: Aspekte zur Diversität der Polychaeta (Annelida) unter besonderer Berücksichtigung der Publikationen von Volker Storch nebst der Beschreibung einer neuen Art der Ampharetida.

Zu Ehren von Volker Storch enthält sein Beitrag die Erstbeschreibung einer neuen Art der Ampharetidae, die auf dem Titelbild dieser Sonderausgabe abgebildet und nach Storch benannt ist.

Mit herzlichen Grüßen und, im Namen aller beteiligten Autoren dieses Sonderheftes, mit einem herzlichen Dank an denjenigen, dem dieser Band gewidmet ist - an Volker Storch, verbleiben wir

Ihr Henner Hollert und Ihr Thomas Braunbeck

\section{Literatur}

Storch V, Wehe T (2007) Biodiversität mariner Organismen: Entstehung - Umfang - Gefährdung. Umweltwiss Schadstoff Forsch 19:213-218 